\title{
Derivação com veias de membro superior após trombólise de aneurisma de artéria poplítea: alternativa para salvamento de membro
}

\author{
Arm vein bypass after popliteal artery aneurysm thrombolysis: \\ an alternative for limb salvage

\begin{abstract}
João Antonio Corrêa ${ }^{1}$, Maria Carolina Cozzi Pires de Oliveira Dias², Alexandre César Fioretti ${ }^{1}$, Yumiko Regina Yamazaki', João Paulo Maffei Jr. ${ }^{1}$, Rogério Duque de Almeida1,
\end{abstract} \\ Fabio Roberto Batistela1, Ohannes Kafejian ${ }^{3}$
}

\section{Resumo}

Os autores relatam um caso de aneurisma de artéria poplítea trombosado em que se realizou fibrinólise com sucesso na fase aguda. Foram utilizadas veias de braço para realização do enxerto e exclusão do aneurisma, pois o paciente havia sido previamente submetido à safenectomia bilateral e revascularização do miocárdio com as veias do outro braço. Apesar das dificuldades, o salvamento do membro foi alcançado. braço.

Palavras-chave: Aneurisma, artéria poplítea fibrinólise, veias de

\section{Introdução}

$\mathrm{O}$ aneurisma de artéria poplítea é o mais freqüente dentre os periféricos ${ }^{1-4}$, sendo atualmente de etiologia multifatorial ${ }^{2}$. Acomete quase que exclusivamente homens, em média acima dos $70 \operatorname{anos}^{1-4}$ e está associado a doença aneurismática da aorta abdominal. A maioria dos pacientes é assintomática e, quando há sintomas, o quadro de isquemia aguda é o mais freqüente. A causa da isquemia pode ser embolia distal ou por trombose da luz aneurismática ${ }^{1,2}$. Outras formas menos comuns de manifestações clínicas seriam os sintomas compressivos, a tumoração em cavo poplíteo e a ruptura.

\begin{abstract}
The authors report a case of a thrombosed popliteal artery aneurysm successfully treated by fibrinolysis in its acute stage. Arm veins were used to perform a bypass and aneurysm exclusion, since the patient had previously been submitted to bilateral saphenous vein stripping and myocardial revascularization using the veins of the other arm. Despite the difficulties, limb salvage was achieved.
\end{abstract}

Keywords: Aneurysm, popliteal artery, fibrinolysis, arm veins.

A trombose aguda freqüentemente causa isquemia crítica, com risco de perda do membro, sendo necessária pronta intervenção para que se obtenha sucesso terapêutico.

\begin{abstract}
Relato de caso
Paciente do sexo masculino, de 78 anos, deu entrada em nosso serviço com quadro clínico de insuficiência arterial aguda, diagnosticada através de exame clínico e cuja arteriografia mostrou ser devida à trombose aguda de aneurisma de artéria poplítea e de artérias de perna (Figura 1A). Foi submetido à trombólise com estrepto-
\end{abstract}

1. Colaboradores, Disciplina de Angiologia e Cirurgia Vascular, Faculdade de Medicina do ABC, Santo André, São Paulo, SP.

2. Residente, Disciplina de Angiologia e Cirurgia Vascular, Faculdade de Medicina do ABC, Santo André, São Paulo, SP.

3. Professor titular, Disciplina de Angiologia e Cirurgia Vascular, Faculdade de Medicina do ABC, Santo André, São Paulo, SP.

Artigo submetido em 19.09.06, aceito em 30.04.07. 
quinase, utilizando inicialmente a técnica de pulse spray com $750.000 \mathrm{U}$, obtendo-se a recanalização parcial da artéria poplítea e das artérias da perna. Após o controle arteriográfico (Figura 1B), optou-se por manter infusão contínua de $5.000 \mathrm{U} / \mathrm{h}$ por $6 \mathrm{~h}$. Obteve-se sucesso terapêutico com a desobstrução da artéria tibial posterior até o arco plantar (Figura 1C).
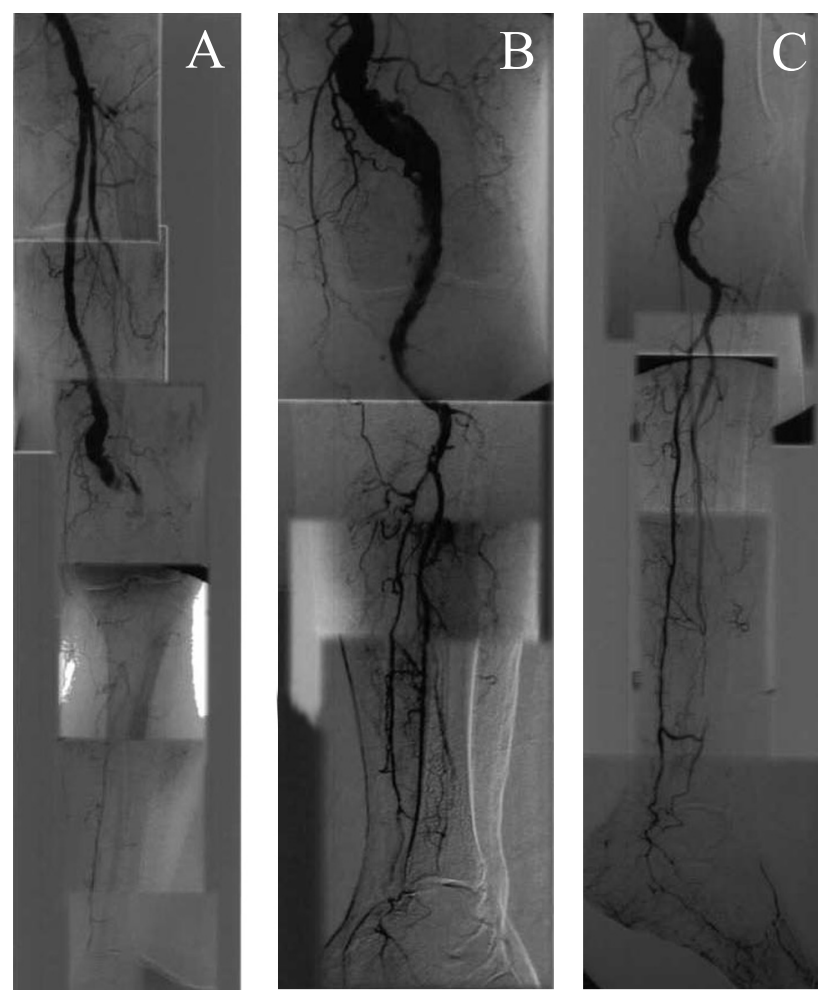

Figura 1 - A) Arteriografia diagnóstica B) após infusão de $750.000 \mathrm{U}$ de estreptoquinase e C) após 6 h de estreptoquinase contínua

O paciente havia sido submetido previamente à safenectomia bilateral por varizes e à ressecção de veias do membro superior esquerdo para revascularização do miocárdio. O tratamento de escolha foi a técnica de exclusão, seguida de revascularização fêmoro-poplítea, por acesso ântero-medial (Figuras 3 e 4), optando-se pela utilização de veia cefálica de antebraço e veia basílica de braço direito, como substituo arterial (Figura 2).

\section{Discussão}

O diagnóstico dos aneurismas da artéria poplítea é feito pelo exame físico associado a exames de imagem,
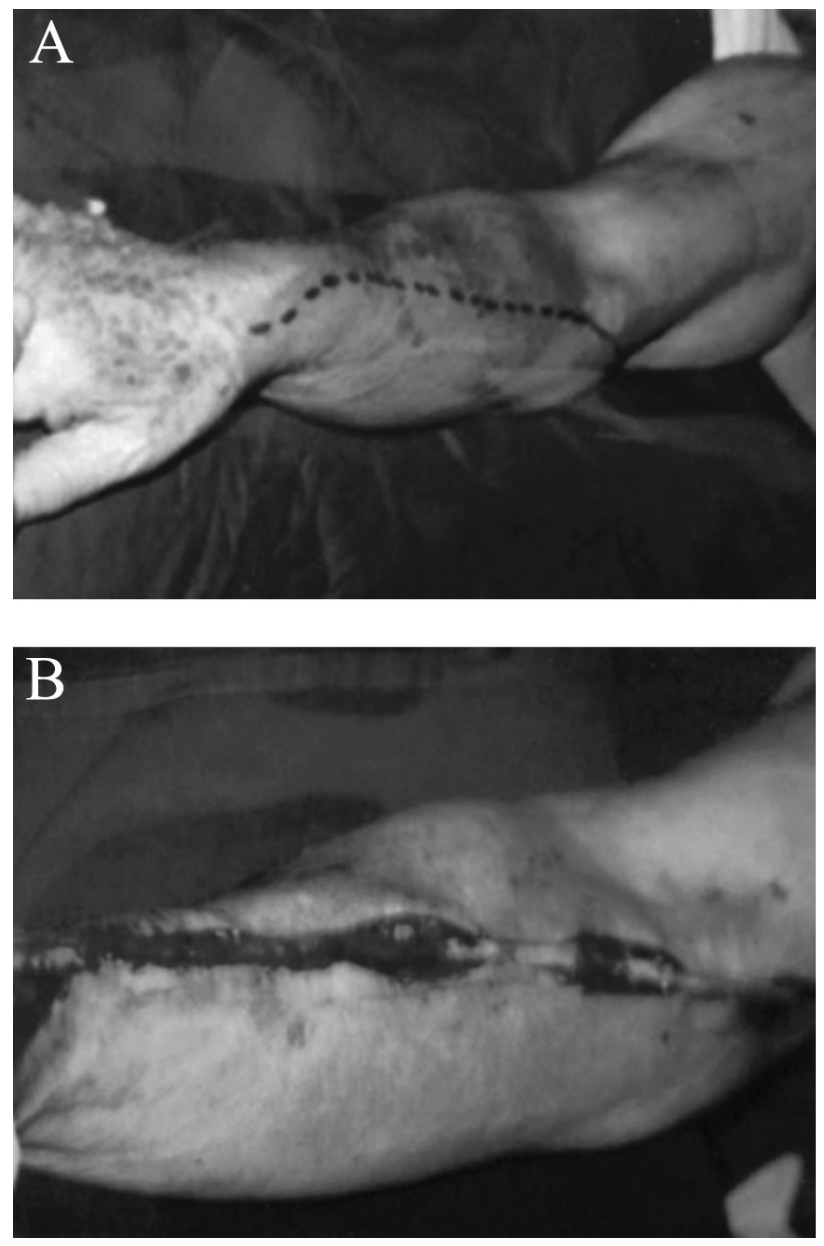

Figura 2 - A) Demarcação e B) veias do antebraço dissecadas

como duplex scan, tomografia computadorizada com contraste ou ressonância nuclear magnética ${ }^{1,2}$. A arteriografia não é exame de escolha para o diagnóstico de aneurisma, porém pode mostrar indícios e orientar a conduta terapêutica.

O tratamento cirúrgico está indicado em casos sintomáticos ou em aneurismas maiores que dois centímetros, com presença de trombo mural ou pobre escoamento distal (sinal de embolização) ${ }^{1-4}$.

A isquemia crítica ocorre em 17 a $46 \%$ dos pacientes com aneurisma de poplítea ${ }^{2}$, sendo que um fator de risco importante para perda do membro é a embolização distal ${ }^{2}$. O escoamento (runoff) fica prejudicado, comprometendo a viabilidade e perviedade do enxerto ${ }^{2}$. Frente à trombose aguda, na isquemia crítica os índices 

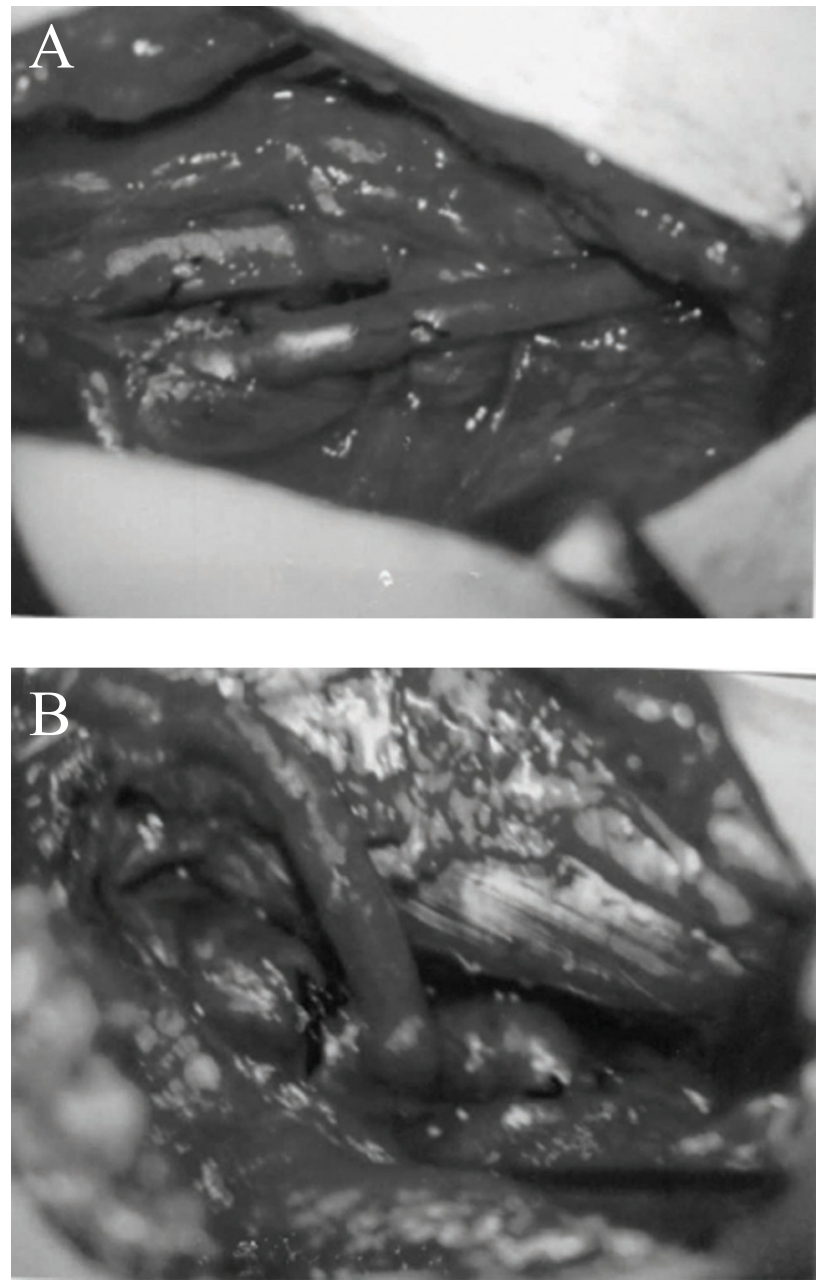

Figura 3 - Anastomose A) proximal e B) distal

de amputações maiores e menores costumam ser elevados, a despeito da revascularização, ocorrendo em 20 a $40 \%$ dos pacientes ${ }^{1,2}$.

O estudo angiográfico está indicado nos casos de trombose aguda do aneurisma de artéria poplítea ${ }^{4}$. Havendo oclusão distal, pode-se indicar a terapia fibrinolítica direcionada por cateter, com a intenção de restaurar a perviedade das artérias de perna e do arco plantar $^{4}$.

Pacientes submetidos à fibrinólise pré-operatória apresentam, após a revascularização, taxas de salvamento de membro de 73 a $100 \%$ em algumas séries ${ }^{2}$. Este sucesso está relacionado à otimização do escoamento distal.
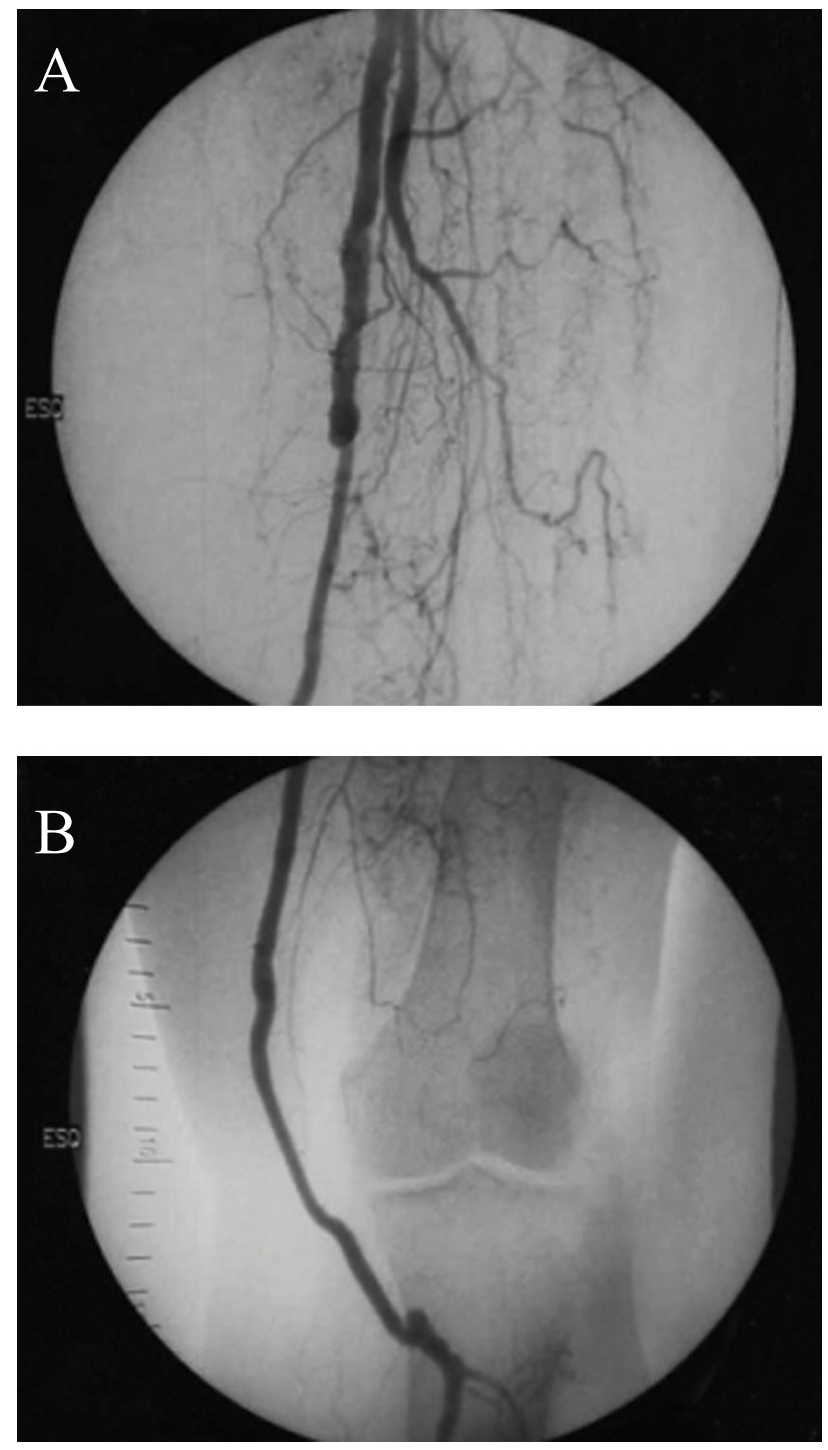

Figura 4 - Anastomose A) proximal e B) distal

O tratamento com fibrinolítico deve ser considerado em casos isquêmicos quando houver tempo hábil para a ação do mesmo, sem colocar o membro em risco ${ }^{3,4}$. A trombólise tem por objetivo recanalizar o leito distal para que se possa realizar posteriormente a revascularização do membro. Obtém-se sucesso quando pelo menos uma artéria de perna é recanalizada até o pé3. Nos casos em que a isquemia é grave e haja necessidade de cirurgia imediata, pode-se realizar a trombólise no intra-operatório, em doses menores ${ }^{5}$. Em pacientes com isquemia crítica e com condição clínica desfavorável, é preferível a amputação primária, pois a tentativa de revascularização apresenta alta morbimortalidade ${ }^{3,5}$. 
Além disso, a terapêutica fibrinolítica apresenta complicações, principalmente hemorrágicas e anafiláticas ${ }^{5}$.

A trombólise, nos casos de aneurisma de artéria poplítea, apenas resolve o evento agudo, sendo necessário o tratamento do aneurisma propriamente dito. Uma das técnicas descritas para sua correção é a exclusão seguida de derivação.

Veias autólogas são consideradas a escolha como substitutos arteriais em derivações infrainguinais ${ }^{6}$. A veia safena interna apresenta índices de salvamento de membro e perviedade incomparáveis, além de menores taxas de infecção ${ }^{6,7}$. Porém, sua falta ou inadequação pode atingir índices de 40 a $45 \%{ }^{6}$. Nestes casos, a utilização de veias autólogas alternativas como as veias safena parva, cefálica, basílica ou femorais profundas podem ser usadas preferencialmente aos substitutos sintéticos, como o politetrafluoretileno expandido (PTFE) e o Dacron ${ }^{\circledR 6}$, com bons resultados ${ }^{1,2}$. Modernamente, o tratamento endovascular figura entre as opções de tratamento, porém os aneurismas de artéria poplítea localizam-se, na maioria das vezes, na área articular do joelho, limitando suas indicações ${ }^{8}$.

Alguns autores preferem o uso das veias de membros superiores ao uso da safena parva pela facilidade de acesso e possibilidade de trabalho em duas equipes, o que diminui o tempo operatório, além de minimizar o número de incisões no membro isquêmico. Além disso, a extensão da veia no membro superior pode ser maior devido à possibilidade de se utilizar a veia cefálica e basílica unidas pela mediana do cotovelo. Pode-se ainda confeccionar anastomoses veno-venosas, sem prejuízo do resultado a longo prazo ${ }^{6}$. É aconselhável o mapeamento venoso prévio com duplex scan, para determinar a qualidade das veias e a melhor opção a ser tomada no ato operatório?.

O seguimento pós-operatório deve ser minucioso, com vigilância do enxerto por duplex scan. Os índices de estenoses e dilatações aneurismáticas são maiores nos enxertos com veias de membros superiores, sendo necessários procedimentos intervencionistas para manutenção da perviedade primária e secundária em até metade dos casos, conforme série norte-americana ${ }^{9}$.

\section{Referências}

1. Aulivola B, Hamdan AD, Hile CN, et al. Popliteal artery aneurysms: a comparison of outcomes in elective versus emergent repair. J Vasc Surg. 2004;39:1171-7.

2. Marty B, Wicky S, Ris HB, et al. Success of thrombolysis as a predictor of outcome in acute thrombosis of popliteal aneurysms. J Vasc Surg. 2002;35:487-93.

3. Illig KA, Eagleton MJ, Shortell CK, Ouriel K, DeWeese JA, Green RM. Ruptured popliteal aneurysm. J Vasc Surg. 1998;27:783-7.

4. Stone PA, Armstrong PA, Bandyk DF, et al. The value of duplex surveillance after open and endovascular popliteal aneurysm repair. J Vasc Surg. 2005;41:936-41.

5. Working Party on Thrombolysis in the Management of Limb Ischemia. Thrombolysis in the management of lower limb peripheral arterial occlusion - a consensus document. J Vasc Interv Radiol. 2003;14(9 Pt 2):S337-49.

6. Faries PL, Arora S, Pomposelli FB Jr., et al. The use of arm vein in lower- extremity revascularization: results of 520 procedures performed in eight years. J Vasc Surg. 2000;31(1 Pt 1):50-9.

7. Albers M, Romiti M, Brochado-Neto FC, Pereira CA. Meta-analysis of alternate autologous vein bypass grafts to infrapopliteal arteries. J Vasc Surg. 2005;42:449-55.

8. Curi MA, Geraghty PJ, Merino OA, et al. Mid-term outcomes of endovascular popliteal artery aneurysm repair. J Vasc Surg. 2007;45:505-10.

9. Armstrong PA, Bandyk DF, Wilson JS, Shames ML, Johnson BL, Back MR. Optimizing infrainguinal arm vein bypass patency with duplex ultrasound surveillance and endovascular therapy. J Vasc Surg. 2004;40:724-30; discussion $730-1$.

Correspondência:

Maria Carolina Cozzi Pires de Oliveira Dias

Rua Professor Moniz, 54, Alto de Pinheiros

CEP 05462-040 - São Paulo, SP

Tel.: (11) 8326.8310

E-mail: carolcozzi@hotmail.com 